\title{
Modification des pratiques d'enseignement de l'EPS : impacts de l'implémentation de formes de pratique scolaire aux secondaires I et II
}

\author{
Yves Suter, Lionel Saillen et Benoît Lenzen
}

\begin{abstract}
Yves Suter.
Lionel Saillen.

Benoît Lenzen.
\end{abstract}

Résumé. En Suisse romande, l'introduction d'un nouveau plan d'études visant, chez l'élève, le développement de compétences, implique une approche nouvelle de la part des enseignants en EPS. C'est sur la base de ce constat qu'un groupe de chercheurs-formateurs s'est employé à analyser ce que peut impliquer un tel type d'approche en EPS, puis à suivre les différentes étapes de l'appropriation de formes de pratiques scolaires (FPS) répondant à de telles approches, par des enseignants, à différents niveaux de la scolarité. Dans la présente étude, l'analyse porte essentiellement sur les expériences menées par 12 enseignants auprès d'élèves de 15 et de 18 ans, ainsi que sur les processus et degrés d'appropriation par ces enseignants des FPS proposées.

Mots-clés. Compétences, Tâche complexe, Évaluation, Appropriation, Diffusion

\section{Introduction}

Depuis 1980, le concept de compétence a pris une place centrale dans les discours, tant institutionnels que professionnels, liés au monde de l'éducation. Pourtant, les approches par compétences se heurtent encore à de multiples obstacles, résultant de la définition "perméable» de la notion même de compétence (Delignières \& Gottsmann, 2016), de différentes difficultés d'appropriation par les enseignants face à toute innovation pédagogique (Vandevelde \& Amade-Escot, 2003) et de divers types de résistances, liées aux institutions et aux programmes scolaires, aux équipes d'enseignants ou encore aux individus (Lenzen, 2012). Dans ce contexte, l'introduction d'une approche par compétences constitue un défi conséquent pour tous les enseignants (Chauvigné \& Coulet, 2010). Et les enseignants en EPS (spécialistes ou non) n’y échappent pas.

En Suisse romande, l'école obligatoire a vécu, durant la dernière décennie, l'introduction d'un nouveau plan d'études, le PER (plan d'études romand), qui vise avant tout, chez l'élève, le développement de compétences. Dès lors, interpellés par les implications que l'introduction d'une telle approche par compétences pouvait avoir sur le plan de l'enseignement et de l'évaluation en EPS, dix chercheurs-formateurs en EPS, provenant de cinq structures de formation des enseignants en Suisse romande, se sont réunis pour former le groupe de recherche PEREPS, dont la démarche s'est appuyée sur les constats et choix suivants :

1. Au vu du contexte de l'étude, le groupe PEREPS a considéré la pertinence d'une approche par compétence en EPS et a retenu la définition du concept de compétence proposé par le PER : «possibilité, pour un individu, de mobiliser un ensemble intégré de ressources en vue d'exercer efficacement une activité considérée généralement comme complexe » 1 . En outre, il a précisé qu'une tâche complexe pouvait être entendue comme « une tâche mobilisant chez l'élève un ensemble de ressources internes (connaissances, savoir-faire, attitudes) et externes (document, fiche technique, matériel, aide méthodologique) qui permet l'expression d'une compétence $» \underline{2}$.

2. Afin de cerner les enjeux liés à l'introduction du PER, le groupe PEREPS s'est intéressé aux attentes fondamentales du PER, en EPS, à chaque niveau d'enseignement. Il a ainsi élaboré un référentiel de compétences pour les niveaux d'enseignement que recouvre le PER : cycle 1 (élèves de 4 à 8 ans), cycle 2 
Modification des pratiques d'enseignement de l'EPS : impacts de l'implémentation de fo...

(9-12 ans) et cycle 3 (ou secondaire I : 13-15 ans). En outre, il a prolongé ce travail au niveau du cycle 4 (ou secondaire II : 16-19 ans).

3. Observant la difficulté à engager les élèves dans un cycle de demi-fond et la nécessité de renouveler l'enseignement de cette APSA (Adé, Jourand \& Sève, 2010 ; Lab, 2004), le groupe a retenu, dans le PER et dans son référentiel de compétences, un axe (condition physique et santé) et un domaine (course de durée) pour approfondir sa réflexion.

4. Pour cibler son processus de recherche, le groupe PEREPS a décidé d'opérer via des formes de pratiques scolaires (FPS) spécifiques (Mascret, 2010), répondant aux compétences attendues à la fin des différents cycles d'enseignement. Il a donc élaboré des FPS pour chaque cycle (Bergé, 2007 ; Hanula, Peyre \& Saulnier, 2015 ; Lab, 2004 ; Millet, 2006), puis il les a testées et fait évoluer de manière empirique. Enfin, il a expérimenté ces FPS auprès d'enseignants volontaires.

L'intérêt du groupe PEREPS a surtout porté sur deux points. D’abord, le groupe souhaitait observer comment des enseignant.e.s s'y prennent pour s'approprier une nouvelle FPS en course de durée et pour construire, à partir de celle-ci, les compétences chez les élèves sur la durée d'un cycle d'enseignement. Ensuite, il s'agissait d'identifier chez les enseignants les besoins en termes d'outils, de formation, de mesures d'accompagnement nécessaires à la mise en place d'une approche de l'enseignement par compétence en EPS.

De manière plus spécifique, la présente étude s'attelle à préciser comment se sont traduites ces différentes questions pour 12 enseignants, aux secondaires 1 et 2.

\section{Méthodologie}

Pour la course de durée, les compétences attendues (selon le référentiel PEREPS) varient en fonction du cycle d'enseignement concerné. Et les FPS qui s'y rapportent proposent divers enjeux d'apprentissage adaptés à chaque cycle, par exemple : respecter, voire enchaîner différentes vitesses de course ; connaître sa VMA et préparer un plan de course adapté ; construire des indices extéro/proprioceptifs pour réguler son allure ; améliorer sa technique de course et sa respiration pour s'économiser ; organiser et coordonner un peloton ; etc.

Au cycle 3, la FPS implique une course continue de 15', séquencée en 10 tranches de 1'30'. Il s'agit d'abord de construire et de gérer un projet de course en peloton constitué de 3-4 coureurs de même potentiel aérobie pour respecter trois vitesses de courses différentes : les élèves doivent élaborer un programme et enchaîner une phase 1 à 60-70\% de leur VMA (tranches 1-2 + ?), puis une phase 2 à 80-90\% de leur VMA (tranches ? + 7-8) en peloton. Il s'agit ensuite d'établir une performance individuelle en fin de course : la phase 3 (tranches 9-10) se déroule individuellement, à une VMA 100\%, que chaque élève doit aussi programmer. Des élèves observateurs prélèvent des données sur la course de leurs condisciples.

$\mathrm{Au}$ cycle 4, il faut concevoir et réaliser un projet de course adapté à sa VMA pour réussir la performance planifiée sur une distance cumulée de 3'000 m (répartie en trois courses de 600, 1'000 et 1'400 m) tout en respectant son plan de course. Ici, la FPS demande à l'élève de préparer individuellement un projet de course en accord avec ses motivations (performance, entraînement, santé) et avec ses possibilités de maintenir une ou des allures différentes (à 75-84\%, à 85-94\%, à 95-100\% ou au-delà de sa VMA), adaptées aux distances de course et à des temps de récupération définis (de 2 à 6 ' selon les allures retenues). De même, les élèves occupent alternativement un rôle de coureur et d'observateur.

Confrontés à ces nouvelles FPS, les enseignants ont librement élaboré, puis animé un cycle d'enseignement : ils ont ainsi construit (ou repris) différents types de tâches pour leurs élèves et ont déterminé certains aspects liés à l'évaluation des compétences acquises par les élèves. Quant au groupe PEREPS, il a mené une phase expérimentale 
Actes de la 11ème Biennale de l'ARIS : Former des citoyens physiquement éduqués. Axe 2 - Vers une éducation physique de qualité, 10.25518/sepaps20.346

articulée en cinq étapes : une information aux enseignants (présentation du contexte général de l'étude et de la FPS les concernant); une phase d'observation (brefs retours à chaud et capture vidéo de quatre leçons); un entretien intermédiaire d'auto-confrontation, appuyé sur des séquences vidéos choisies par un chercheur ; une récolte de traces didactiques (planifications, fiches d'observation...) ; un entretien post-expérimental, de type semi-directif.

Dans le contexte d'une démarche d'ingénierie didactique (Artigue, 1989), l'intérêt du groupe PEREPS s'est essentiellement porté sur les trois temps du processus d'appropriation des FPS proposées : l'élaboration, la réalisation et l'analyse a posteriori du projet didactique. Dans le cadre de cette contribution, nous avons analysé, puis comparé, les démarches d'appropriation planifiées et effectivement entreprises par les enseignants. Nous nous sommes aussi focalisés sur les entretiens enregistrés. Nous avons finalement porté notre intérêt sur les conflits intrapsychiques qui découlent de ce type d'expérimentation chez les enseignants (Vygotsky, 1997), ainsi que sur le recoupement ou non entre discours déclaratifs et comportements observés.

\section{Résultats et discussion}

L'analyse des démarches d'appropriation initiales (canevas de leçons et autres documents) met en relief les constats suivants :

1. La présentation de la FPS aux élèves est effectuée dès le début du cycle d'enseignement dans neuf cas sur douze, tandis que trois enseignants n'abordent la FPS que plus tard. Quant aux références à la FPS, elles sont très inégales au cours des cycles d'enseignement.

2. Les projets de travail reposent (assez) fréquemment sur la régularité de la course et sur les différentes allures de course. Mais les projets s'intéressent moins souvent à la notion de respiration/essoufflement. Et le travail sur la réelle construction d'indices pour réguler ses allures de course (fréquence des foulées, appuis, observation du peloton, etc.) est peu présent. Par conséquent, si les types de tâches orientés vers la FPS sont assez fréquents, les tâches avec aménagements et contraintes ciblés sur une habileté motrice précise pour faciliter la compréhension ou la réalisation de certaines facettes de la FPS sont plus rares.

3. Sur le plan de l'évaluation, tous les enseignants ont mis un barème en place, mais de formes assez différentes. On relèvera notamment que, chez deux enseignants, le barème était très clairement codifié... tandis qu'il y avait au moins un flou et/ou une omission chez huit autres enseignants. Et l'on notera que quatre enseignants ont adjoint un test pur de performance (test de Cooper, course de $8 \mathrm{~km} . .$. ) à leur évaluation.

L'étude des leçons filmées, associée à celle des synopsis qui en découlent, permet de reprendre les constats initiaux, mais pointe des caractéristiques sensiblement différentes selon qu'il s'agit de l'un ou de l'autre cycle d'enseignement :

1. Au cycle 3, la FPS (adaptée ou non) est très présente chez cinq enseignants, mais un peu moins chez le dernier. Tous les enseignants traitent les allures de courses, quatre enseignants abordent les questions de respiration et travaillent un peu le développement de la puissance aérobie. Trois enseignants recherchent divers indices d'aide avec leurs élèves, alors que deux enseignants ne traitent pas cet objet. Cinq enseignants prennent du temps pour aborder la gestion du peloton. Enfin, deux enseignants ont un discours orienté « réflexivité » (mise en perspective, aide à l'appropriation...), tandis que trois enseignants s'y essaient. Mais ces discours portent peu sur l'activité des observateurs.

2. Au cycle 4, la FPS (adaptée ou non) est très présente chez trois enseignants, mais moins chez les trois autres. Tous les enseignants insistent sur les allures de courses, traitent les questions de respiration et travaillent la capacité aérobie. Quatre enseignants cherchent divers indices d'aide (deux d'entre eux le font même en profondeur), tandis que trois enseignants travaillent sur les plans de course (les trois autres ne 
Modification des pratiques d'enseignement de l'EPS : impacts de l'implémentation de fo...

traitant quasiment pas cet enjeu d'apprentissage). Finalement, quatre enseignants ont un discours orienté « réflexivité » et, pour deux d'entre eux, aussi vis-à-vis des observateurs.

En général, les entretiens d'auto-confrontation (EAC) menés à mi-parcours ont eu peu d'impact sur la suite de l'enseignement. D'un côté, chez certains enseignants, ces EAC n'ont pas mis en évidence de problème fondamental, tandis que, chez d'autres, la planification du cycle d'apprentissage était déjà finalisée (et ces enseignants ne voulaient pas revenir sur un travail déjà lourd d'investissement). D’un autre côté, pour deux enseignants, ces EAC ont permis la prise de conscience d'un décalage entre intentions et actions effectives, alors que, chez deux autres enseignants, ces EAC ont confirmé un besoin de changement (jusqu'alors largement pressenti).

Pour terminer, l'analyse des entretiens semi-directifs a mis en lumière une réalité, une série de difficultés et d'interrogations, enfin certains besoins :

1. Pour la plupart des enseignants, le changement dans l'approche est patent. D'une part, le travail en endurance est modifié : dix enseignants relèvent l'intérêt des élèves à construire des allures de course, six enseignants mentionnent spontanément la pertinence du travail autour de la prise de repères (surtout au niveau de la respiration), tandis que certains soulignent l'intérêt de la collaboration entre élèves, de l'autonomisation des élèves ou encore de l'individualisation du travail pour les élèves. D’autre part, cela suscite une réflexion générale intéressante et un questionnement personnel chez les enseignants, qui mentionnent souvent que ce questionnement est encore (grandement) en cours.

2. L'appropriation de ces FPS n'a pas toujours été facile. Ainsi, sept enseignants relèvent un travail supplémentaire (conséquent), voire du stress: pour s'approprier le projet, pour en cerner les enjeux, pour planifier/préparer l'enseignement ou pour créer un enseignement personnalisé d'une leçon à l'autre. Par ailleurs, certains enseignants (surtout au cycle 3) relèvent des difficultés pratiques et un besoin d'adaptations : problème de distinction marche/course pour des VMA très basses ; modification nécessaire de la FPS en fonction des conditions matérielles; projets complexes et difficiles à gérer (surtout pour les élèves en difficulté scolaire) ; problématiques liées au contrôle et à de possibles tricheries.

3. Malgré ces bémols, l'intérêt pour de tels projets semble clair, puisque 11 enseignants sont à priori favorables à une reprise de cette FPS ou d'autres FPS de ce type. Cela permet une évolution des pratiques : c'est complexe (et stimulant). On est orienté/aidé (documents). Il y a des libertés (construction, barème). La rigueur des allures à tenir constitue un enjeu pertinent. Enfin, pour le cycle 3, l'idée de peloton rencontre une forte adhésion.

4. Malgré l'intérêt des 12 enseignants concernés, une pérennisation de ce type d'approche n'est pas garantie. Premièrement, pour un élargissement au sein d'un établissement scolaire, il y a un besoin d'adhésion généralisée au projet. Deuxièmement, la transposition vers d'autres sports (collectifs ou autres) semble délicate : difficulté à distinguer les enjeux d'apprentissage, trop grande complexité, etc. Troisièmement, il y a des besoins en termes de formation: dans le cas présent, dix enseignants auraient souhaité plus d'informations et huit d'entre eux sont d'avis qu'une formation continue, au départ, serait un plus. Quatrièmement, ces besoins en formation sont inégaux, tant en quantité qu'en qualité.

\section{Conclusions et perspectives}

Comment des enseignants s'y prennent-ils pour s'approprier une nouvelle FPS et pour construire, à partir de celle-ci, les compétences chez les élèves durant un cycle d'enseignement? Force est de constater que les degrés d'appropriation dépendent largement de l'épistémologie pratique des enseignants (Amade-Escot, 2019). Par ailleurs, les contraintes 
Actes de la 11ème Biennale de l'ARIS : Former des citoyens physiquement éduqués. Axe 2 - Vers une éducation physique de qualité, 10.25518/sepaps20.346

liées à l'établissement dans lequel les enseignants travaillent (programmes, conditions matérielles, types d'élèves ou groupe d'enseignants) ont également une influence.

Quels sont les besoins, chez ces enseignants, en termes d'outils, de formation, de mesures d'accompagnement nécessaires à la mise en place d'une approche de l'enseignement par compétence ? Là aussi, cela dépend des enseignants, chez qui les besoins sont multiples. D'abord, il faut du temps et du travail : pour s'approprier les principes, pour discuter ou pour adapter ses pratiques, etc. Mais ensuite, les enseignants doivent bénéficier d'inputs solides (formation continue, documents complémentaires, aide à l'appropriation « en continu », portant notamment sur la théorie de l'approche par compétences, sur la mise en évidence de points-clés par rapport à de telles FPS, sur des exemples de stratégies à mettre en place et sur des propositions de canevas de cours), ainsi que d'un contexte d'enseignement adéquat (implication d'un établissement scolaire dans son ensemble par exemple).

1 Plan d'études romand, Site du PER : $\underline{\text { https://www.plandetudes.ch/web/guest/pg2-lexique }}$

$\underline{2}$ Définition du groupe de recherche PEREPS

\section{Bibliographie}

Adé D., Jourand C., \& Sève C. (2010). L'inscription contextuelle de l'activité en course en durée : Une étude à partir de l'analyse de l'activité d'élèves de primaire en éducation physique et sportive. Éducation et didactique, 4, 7-22.

Amade-Escot, C. (2019). Epistémologies pratiques et action didactique conjointe du professeur et des élèves. Education et didactique, 13, 109-114.

Artigue, M. (1989). Ingénierie didactique. Recherches en didactique des mathématiques, 9 (3), 281-308.

Bergé, A. (2007). Une forme de pratique scolaire de course de durée. Les cahiers du CEDRE, 7, 53-59.

Chauvigné C., \& Coulet J.-C. (2010) L'approche par compétences: un nouveau paradigme pour la pédagogie universitaire? Revue française de pédagogie, 172, 15-28.

Delignières D., \& Gottsmann L. (2016). À propos des obstacles épistémologiques à l'émergence du concept de compétence. Movement \& Sport Sciences, 94, 71-81.

Hanula, G., Peyre, C., \& Saulnier, J.-Y. (2015). Un itinéraire pour le savoir s'entraîner en CP5 : vers la compétence attendue en niveau 4 pour la course de durée. Les cahiers du CEDRE, 15, 139-153.

Lab, F. (2004). Analyse critique des contenus d'enseignement en course de durée proposés dans les écrits professionnels. eJRIEPS, 5, 37-62.

Lenzen, B. (2012). Les activités curriculaires des enseignants d'EPS, entre prescription et liberté : une revue de littérature. eJRIEPS, 27, 27-65.

Mascret, N. (2010). L'élaboration d'une forme de pratique scolaire d'une APSA en EPS : un geste professionnel complexe. In M. Cizeron \& N. Gal-Petifaux (Eds.), Analyse de pratiques : expérience et gestes professionnels (pp. 193-203). Clermont-Ferrand : Presses Universitaires Blaise Pascal.

Millet, G. (2006). L'endurance. Paris : Editions Revue EP.S.

Vandevelde M. \& Amade-Escot C. (2003). Etude des modalités d'incorporation de savoirs d'expert, dans le cadre de la formation continue en EPS. eJRIEPS, 3, 51-66. 
Modification des pratiques d'enseignement de l'EPS : impacts de l'implémentation de fo...

Vygotsky, L. S. (1997). Thought and language. Paris : La Dispute.

\section{Notes}

1 Plan d'études romand, Site du PER : https://www.plandetudes.ch/web/guest/pg2-lexique

$\underline{2}$ Définition du groupe de recherche PEREPS

PDF automatiquement généré le 2021-10-22 14:07:51

Url de l'article : https://popups.uliege.be/sepaps20/index.php?id=346

Publié par ULiège Library en Open Access et distribué suivant les termes et les conditions de la licence CC-BY

(https://creativecommons.org/licenses/by/4.0/deed.fr) 